\title{
Families of Varieties of General Type: the Shafarevich Conjecture and Related Problems
}

\section{SÁNDOR J. KOVÁCS*}

Consider the curves on the $(x, y)$-plane parametrized by $\lambda$ and given by the equation,

$$
y^{2}=x^{5}-5 \lambda x+4 \lambda .
$$

An easy computation shows that these curves are non-singular for $\lambda \in$ $\mathbb{C} \backslash\{0,1\}$. On the other hand the projective closure of each of these curves is singular at the point $[0: 1: 0]$. Blowing up this point and another one infinitely near this one yields a resolution, $C_{\lambda}$. The collection of $C_{\lambda}$ forms a family of smooth projective curves over the base $\mathbb{C} \backslash\{0,1\}$.

Exercise 0.1. Prove that the projection of $C_{\lambda}$ onto the $y=0$ axis is the only degree 2 morphism onto $\mathbb{P}^{1}$ that $C_{\lambda}$ admits. (Hint: See [28], IV.5.3). Conclude that for $\lambda_{1} \neq \lambda_{2}, C_{\lambda_{1}} \not C_{\lambda_{2}}$.

A family of curves is called isotrivial if any two general members are isomorphic. For example the blow up of the projective plane at a single point, considered as a $\mathbb{P}^{1}$-bundle over $\mathbb{P}^{1}$, is an isotrivial family since all of its members are isomorphic to the projective line. Since the genus of a curve in a family is constant, and there is only one curve of genus zero, one cannot expect much more in this case. However, for higher genus curves one can have non-isotrivial families as the above example shows.

Exercise 0.2. Compute the genus of $C_{\lambda}$. Find another non-isotrivial family of smooth curves of the same genus over $\mathbb{C} \backslash\{0,1\}$. Make a guess: Is there an infinite sequence of pairwise different families of that kind?

At the 1962 ICM in Stockholm, Shafarevich conjectured that there exist only finitely many isomorphism classes of non-isotrivial families of smooth

\footnotetext{
* Supported in part by NSF Grants DMS-0196072, DMS-0092165, and a Sloan Research Fellowship.
} 
projective curves of a given genus over a given base curve. Furthermore, if there is such a family, then the base curve satisfies a certain hyperbolic condition. (For definitions and a more precise formulation, see Section §1). The conjecture was confirmed by Parshin [57] for the case of a compact base and by Arakelov [3] in general. It was recently generalized to families of higher dimensional varieties. This generalization is our main topic.

It will be advantageous to work with a compactification of the family. Considering families over a compact base curve $B$ naturally leads to a slightly different view on the problem. Instead of smooth families over a non-compact base, we are looking at arbitrary families over a compact base, and consider the locus over which the family is smooth. It is fairly obvious that these are equivalent situations. An arbitrary family over a compact base gives a smooth family over some open subset and a smooth family over an open curve can be extended to a (not necessarily smooth) family over the projective closure of the curve cf. [28], III.9.8.

One could ask what can be said about the singular members of the family. On the simplest level, how many are there? In fact, Szpiro has asked this: What is the lower bound on the number of singular members if $B \simeq \mathbb{P}^{1}$ ? This is the same as asking how close the base of the smooth family can be to being compact. Beauville [6] gave the following answer to Szpiro's question: there are always at least three singular members and there are families with exactly three. In fact, Beauville's proof also shows that there is at least one singular member if the base curve is elliptic. In short, $2 g(B)-2+\delta>0$, where $g(B)$ is the genus of the (compact) base curve and $\delta$ is the number of singular members of the family. In other words, the base of a smooth family must be hyperbolic.

Notice that the existence of Kodaira surfaces shows that there are families over high genus curves without any singular fibers. For the construction of Kodaira surfaces see for example [5], V.14. An interesting point to mention is that even though we do not expect general obstacles for higher genus bases, the known examples are limited in terms of the possible base curves. Taking repeated general hyperplane sections in a moduli space of curves, one can produce "examples" over compact curves, however, this still does not produce a method to find a family over a given curve. It would be interesting to see explicit examples that are significantly different from the Kodaira construction. Having said that, we return to the opposite end of the problem, the focus of this article.

Recently, Catanese and Schneider [8] asked whether something similar to the Szpiro-Beauville bound holds for families with higher dimensional 
fibers. The conjecture of Shokurov [59] translates to the same question: Is it true that for a family of varieties of general type, $\delta \geq 3$ if $g(B)=0$ and $\delta \geq 1$ if $g(B)=1$ ? Equivalently, is $2 g(B)-2+\delta>0$ ? In effect we are asking whether "hyperbolicity of the base" holds in higher dimensions.

The wide range of applications this question relates to is interesting to note: Catanese and Schneider wanted to use this statement to obtain good estimates for the size of the automorphism group of a variety of general type, while Shokurov needed it for proving quasi-projectivity of certain moduli spaces. Hyperbolicity for families of curves was used by Moret-Bailly [53] and de Jong and Oort [12] to extend those families over codimension 2 subsets.

The structure of the article is as follows. In the first two sections, the original Shafarevich conjecture is discussed in the function field and in the number field case. Section $\S 3$ is a brief account of hyperbolicity in general. Section $\S 4$ is a short look at a few vanishing theorems. Section $\S 5$ contains a sketch of how to prove hyperbolicity of the base for families of curves. Section $\S 6$ is a short introduction to the theory of moduli of curves. Beginning with Section $\S 7$, we turn to a more detailed discussion of the tools and ideas involved. Sections $\S 7$ and $\S 8$ contain preliminary steps to Section $\S 9$. Weak boundedness, introduced in Section $\S 9$, turns out to be one of the most important notions we encounter. In Section $\S 10$ we turn to higher dimensional generalizations of the Shafarevich problem. Section $\S 11$ contains a brief discussion of positivity of push-forwards and Section $\S 12$ is a tour of more vanishing theorems. Section $\S 13$ deals with a further generalization, which ultimately leads to studying singular families. Section $\S 14$ touches on the subject of families over higher dimensional bases.

Acknowledgements. My work on the subject and consequently this article owes a great deal to János Kollár and Eckart Viehweg. Their ideas and philosophy are present throughout.

I would like to thank Ron Irving, Grisha Stewart, Zack Treisman, and Michael Van Opstall for numerous suggestions that helped improve the presentation of the article.

Finally, I would like to dedicate this article to József Pelikán, whose abstract algebra and topics courses were very influential during my undergraduate years at Eötvös University. 


\section{$\S 1$. The Function Field Case}

The following notation will be preserved throughout the entire article:

Notation 1.1. Let $B$ be a smooth projective curve of genus $g$ over an algebraically closed field $k$ of characteristic 0 and $\Delta \subset B$ a finite subset. In the sequel $B$ and $\Delta$ will be fixed.

We will consider families over $B$, i.e., flat projective morphisms $f$ : $X \rightarrow B$ with connected fibers, where $\mathrm{X}$ is a smooth projective variety over $k$.

For a morphism $Y \rightarrow S$ and a base change morphism $T \rightarrow S$, the symbol $Y_{T}$ will denote $Y \times_{S} T$. In particular, for $Y=X, S=B$ and $b \in B$ we write $X_{b}=f^{-1}(b)$. In addition, if $T=\operatorname{Spec} F$, then $Y_{T}$ will also be denoted by $Y_{F}$.

Definition 1.2. A family $f: X \rightarrow B$ is isotrivial if $X_{a} \simeq X_{b}$ for general points $a, b \in B$. The family $f: X \rightarrow B$ will be called admissible (with respect to $(B, \Delta))$ if it is not isotrivial and $\Delta$ contains the discriminant locus of $f$, i.e., the map $f: X \backslash f^{-1}(\Delta) \rightarrow B \backslash \Delta$ is smooth.

Our starting point is the aforementioned conjecture of Shafarevich:

1.3. Shafarevich's Conjecture. Let $(B, \Delta)$ be fixed and $q \geq 2$ an integer.

(1.3.1) There exist only finitely many isomorphism classes of admissible families of curves of genus $q$.

(1.3.2) If $2 g-2+\# \Delta \leq 0$, then there exist no such families.

Exercise 1.4. Prove that the inequality in (1.3.2) can be satisfied only if $B$ is either a rational or an elliptic curve:

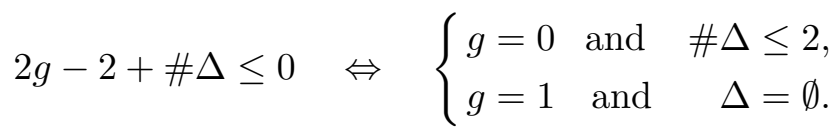

Shafarevich showed a special case of (1.3.2): There exist no smooth families of curves of genus $q$ over $\mathbb{P}^{1}$. (1.3.1) was confirmed by Parshin [57] for $\Delta=\emptyset$ and by Arakelov [3] in general.

Our main goal is to generalize this statement to higher dimensional families. In order to do that we will have to reformulate the statement as Parshin and Arakelov did, but before doing so, we need a little bit of background on deformations and parameter spaces. 
1.5. Deformations. In general, a deformation of an object means to include that object in a family. There is a potentially confusing point here. Our main objects of study are families, that is, deformations of their members. However, we do not want to consider our families as deformations. We want to look at deformations of these families. This may be a bit harder to imagine first, but it works just the same way as deformations of other objects. In addition, we want to fix the base of these families, so we are interested in deformations leaving the base fixed, which makes both the notation and the theory easier.

A deformation of a family $\phi: Y \rightarrow S$ with the base fixed is a family $\mathfrak{Y} \rightarrow S \times T$, where $T$ is connected and such that for some $t_{0} \in T$ we have $\left(\mathfrak{Y}_{t_{0}} \rightarrow S \times\left\{t_{0}\right\}\right) \simeq(Y \rightarrow S):$

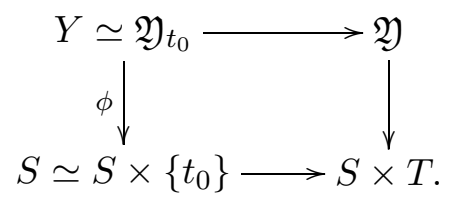

We say that two families $Y_{1} \rightarrow S$ and $Y_{2} \rightarrow S$ have the same deformation type if they can be deformed into each other, i.e., if there exists a connected $T$ and a family $\mathfrak{Y} \rightarrow S \times T$ such that for some $t_{1}, t_{2} \in T$,

$$
\left(\mathfrak{Y}_{t_{i}} \rightarrow S \times\left\{t_{i}\right\}\right) \simeq\left(Y_{i} \rightarrow S\right) \text { for } \quad i=1,2
$$

We will consider deformations of admissible families. It will be advantageous to restrict to deformations of the family over $B \backslash \Delta$. Doing so potentially allows more deformations than over the original base $B$ : it can easily happen that a deformation over $B \backslash \Delta$, that is, a family $\mathfrak{X} \rightarrow(B \backslash \Delta) \times T$, cannot be compactified to a (flat) family over $B \times T$, because the compactification may contain fibers of higher than expected dimension. This however, will not cause any problems because of the nature of our inquiry cf. (1.7).

Parameter Spaces. Given a family $\phi: Y \rightarrow S$, we say that $S$ parametrizes the members of the family. If the members of the family $\phi$ form a class, $\mathfrak{C}$, of varieties, then we say that $S$ is a parameter space for the class $\mathfrak{C}$. Note that we do not require that the members of $\mathfrak{C}$ appear only once in the family.

Exercise 1.6. Show that $\mathbb{P}^{5}$ is a parameter space for plane conics. 
A very useful parameter space is the Hilbert scheme, a parameter space for subschemes of $\mathbb{P}^{n}$. The Hilbert scheme of $\mathbb{P}^{n}$ decomposes as the disjoint union of Hilbert schemes of subschemes with a given Hilbert polynomial. The components of this union are projective schemes (in particular of finite type). When one is hoping to parametrize the members of a class of varieties, then the most likely way to succeed is to try to find the parameter space as a subscheme of an appropriate Hilbert scheme. For more on Hilbert schemes see [37] and [66]. We will return to their importance and some applications in Section $\S 6$.

For more on parameter spaces see [24], Lectures 4, 21.

1.7. With regard to the Shafarevich conjecture, Parshin made the following observation. In order to prove that there are only finitely many admissible families, one can try to proceed the following way. Instead of aiming for the general statement, first try to prove that there are only finitely many deformation types. This is expected to be somewhat easier, because there are ways to parametrize deformations, and the fact that there are only finitely many types translates to the parameter space being of finite type. The next step then is to prove that admissible families are rigid, that is, they do not admit non-trivial deformations. Notice that if we prove these statements for families over $B \backslash \Delta$, then they also follow for families over $B$. Now since every deformation type contains only one family, and since there are only finitely many deformation types, the original statement follows.

The following is the reformulation of the Shafarevich conjecture that was used by Parshin and Arakelov.

1.8. Reformulation. Let $q \geq 2$ be an integer. Recall that $(B, \Delta)$ is fixed.

(1.8.1) Boundedness (B): There exist only finitely many deformation types of admissible families, i.e., admissible families of curves of genus $q$ are parametrized by $\mathbb{T}$, a scheme of finite type.

(1.8.2) $\underline{\text { Rigidity }}(\mathbf{R})$ : There exist no non-trivial deformations of admissible families of curves of genus $q$, i.e., $\operatorname{dim} \mathbb{T}=0$.

(1.8.3) Hyperbolicity $(\mathbf{H})$ : No admissible families of curves of genus $q$ exist if $2 g-2+\# \Delta \leq 0$, i.e., $\mathbb{T} \neq \emptyset \Rightarrow 2 g-2+\# \Delta>0$.

Remark 1.8.4. As we discussed above, $(\mathbf{B})$ and $(\mathbf{R})$ together imply (1.3.1) and $(\mathbf{H})$ is clearly equivalent to (1.3.2). 


\section{§2. The Number Field Case}

The number field version of Shafarevich's conjecture played a very important role in Faltings' proof of the Mordell conjecture. This section is a brief detour to this very exciting area, but it is disconnected from the rest of the article. The reader should feel free to skip this section and continue with the next one.

Definition 2.1. Let $(R, \mathfrak{m})$ be a DVR, $F=\operatorname{Frac}(R)$, and $C$ a smooth projective curve over $F$. $C$ is said to have good reduction over $R$ if there exists a scheme $Z$, smooth and projective over $\operatorname{Spec} R$, such that $C \simeq Z_{F}$,

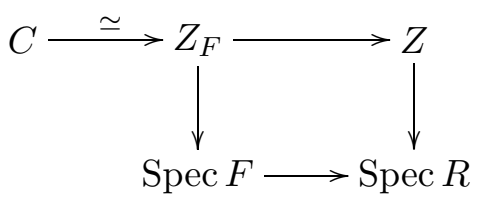

Definition 2.2. Let $R$ be a Dedekind ring, $F=\operatorname{Frac}(R)$, and $C$ a smooth projective curve over $F$. $C$ has good reduction at the closed point $\mathfrak{m} \in \operatorname{Spec} R$ if it has good reduction over $R_{\mathfrak{m}}$.

2.3. Shafarevich Conjecture. Let $q \geq 2$ be an integer.

(2.3.1) Let $F$ be a number field, $R \subset F$ the ring of integers of $F$, and $\Delta \subset \operatorname{Spec} R$ a finite set. Then there exists only finitely many smooth projective curves over $F$ of genus $q$ that have good reduction outside $\Delta$.

(2.3.2) There are no smooth projective curves of genus $q$ over Spec $\mathbb{Z}$.

Remark 2.3.3. The Shafarevich Conjecture in the number field case has been confirmed: (2.3.1) by Faltings [20] and (2.3.2) by Fontaine [21].

One can reformulate (1.3.1) to resemble the above statement:

2.4. Shafarevich Conjecture (function field case, version 2). Let $q \geq 2$ be an integer, $F=K(B)$ the function field of $B$. We may assume that $\Delta$ is not empty, i.e., that $B \backslash \Delta$ is affine. Let $R$ be the subring of $F$ such that $B \backslash \Delta=\operatorname{Spec} R$. Then there exist only finitely many smooth projective non-isotrivial curves over $F$ of genus $q$ having good reduction over all closed points of $\operatorname{Spec} R$. 
Definition 2.5. If $C$ is a smooth projective curve over $F$ (an arbitrary field), then there exists a morphism $C \rightarrow \operatorname{Spec} F$. Sections, Spec $F \rightarrow C$, of this morphism correspond in a one-to-one manner to $F$-rational points of $C$, points that are defined over the field $F$. $F$-rational points of $C$ will be denoted by $C(F)$.

\section{Example 2.5.1.}

(1) The $\mathbb{R}$-rational points of the curve $x^{2}+y^{2}-z^{2}=0$ form a circle, its $\mathbb{C}$-rational points form a sphere.

(2) The curve $x^{2}+y^{2}+z^{2}=0$ has no $\mathbb{R}$-rational points.

(3) Let $C_{n}$ be the curve defined by the equation $x^{n}+y^{n}-z^{n}=0$. If $n \geq 3$, then according to Wiles' Theorem, which is also known as Fermat's Last Theorem,

$$
C_{n}(\mathbb{Q})= \begin{cases}\{[1: 0: 1],[0: 1: 1],[1:-1: 0]\}, & \text { if } n \text { is odd } \\ \{[1: 0: 1],[0: 1: 1],[1: 0:-1],[0: 1:-1]\} & \text { if } n \text { is even }\end{cases}
$$

As mentioned earlier, Faltings used (2.3) to prove:

2.6. Mordell Conjecture. Let $F$ be a number field and $C$ a smooth projective curve of genus $q \geq 2$ defined over $F$. Then $C(F)$ is finite. [51]:

The function field version of this conjecture was proved earlier by Manin

2.7. Mordell Conjecture for function fields. Let $F$ be a function field (i.e., the function field of a variety over $k$, where $k$ is an algebraically closed field of characteristic 0 ) and let $C$ be a smooth projective non-isotrivial curve over $F$ of genus $q \geq 2$. Then $C(F)$ is finite.

Remark 2.7.1. The essential case to settle is when $\operatorname{tr} \cdot \operatorname{deg}_{k} F=1$, i.e., $F=K(B)$, where $B$ is a smooth projective curve over $k$.

Exercise 2.8. Let $B$ be a smooth projective curve over $k$ and $F=K(B)$ its function field. Let $C$ be a smooth projective curve over $F$, and let $f$ : $X \rightarrow B$ be the "closure" of $f_{0}: C \rightarrow$ Spec $F$. Prove that the following statements are equivalent.

(1) There exists an $F$-rational point of $C$;

(2) There exists a Spec $F$-section of $f_{0}$;

(3) There exists a $B$-section of $f$. 
Shafarevich's conjecture implies Mordell's in both the function field and the number field case by an argument due to Parshin:

2.9. Parshin's Covering Trick. For every $P \in C(F)$ or equivalently, for every section $X \stackrel{\sigma_{P}}{\rightarrow} B$, there exists a finite cover of $X, W_{P} \stackrel{\pi_{P}}{\longrightarrow} X$ such that

- the degree of $\pi_{P}$ is bounded in terms of $q$,

- the projection $W_{P} \rightarrow B$ is smooth over $B \backslash \Delta$,

- the map $\pi_{P}$ is ramified exactly over the image of $\sigma_{P}$,

- the genus of the fibers of $W_{P} \rightarrow B$ is bounded in terms of $q$.

For details on the construction of the covers, $W_{P} \stackrel{\pi_{P}}{\longrightarrow} X$, see [50], IV.2.1.

The last ingredient of the proof is:

Theorem 2.10. (de Franchis [11]) Let $C$ and $D$ be smooth projective curves of genus at least two. Then there exist only finitely many dominant rational maps $D \rightarrow C$.

The Shafarevich Conjecture implies that there are only finitely many different $W_{P}$ 's. Viewing $W_{P}$ and $X$ as curves over $F$, de Franchis' theorem implies that a fixed $W_{P}$ can admit only finitely many different maps to $X$.

Since those maps are ramified exactly over the image of the corresponding $\sigma_{P}$, this means that there are only finitely many $\sigma_{P}$ 's, i.e., $C(F)$ is finite, and therefore Mordell's conjecture follows from that of Shafarevich.

We end our little excursion to the number field case here. In the rest of the article we work in the function field case and use the notation and assumptions of (1.1).

\section{§3. HYPERBOLICITY}

Definition 3.1. A complex analytic space $X$ is called Brody hyperbolic if every holomorphic map $\mathbb{C} \rightarrow X$ is constant.

Remark 3.1.1. Another important, related notion is Kobayashi hyperbolicity. For its definition and relation to Brody hyperbolicity the reader is referred to [49]. 
Exercise 3.2. Let $T$ be a complex torus. Prove that if $\mathrm{X}$ is Brody hyperbolic, then every holomorphic map $\mathbb{C}^{*} \rightarrow X$ is constant, and every holomorphic map $T \rightarrow X$ is constant.

We would like to define the algebraic analogue of hyperbolicity motivated by this observation. Algebraic maps are more restrictive than holomorphic ones. For instance the universal covering map, $\mathbb{C} \rightarrow E$, of an elliptic curve, $E$, is not algebraic. In particular, excluding algebraic maps from $\mathbb{C}$ to $X$ does not exclude maps from $E$ to $X$.

The following is a working definition. For a notion that resembles Kobayashi hyperbolicity one would need a different definition. This should perhaps be called "algebraic Brody hyperbolicity" to emphasize that fact. Nevertheless, the following is the way it is currently used:

Definition 3.3. An algebraic variety $X$ is called algebraically hyperbolic if

- every regular map $\mathbb{A}^{1} \backslash\{0\} \rightarrow X$ is constant, and

- every regular map $A \rightarrow X$ is constant, where $A$ is an abelian variety.

Next we recall some basic, but very important notions.

Definition 3.4. Let $\mathcal{L}$ be a line bundle on $X$. It is said to be generated by global sections if for every point $x \in X$ there exists a global section $\sigma_{x} \in H^{0}(X, \mathcal{L})$ such that the germ $\sigma_{x}$ generates the stalk $\mathcal{L}_{x}$ as an $\mathcal{O}_{X^{-}}$ module. If $\mathcal{L}$ is generated by global sections, then the global sections define a morphism $\phi_{\mathcal{L}}: X \rightarrow \mathbb{P}^{N}=\mathbb{P}\left(H^{0}(X, \mathcal{L})\right) . \mathcal{L}$ is called semi-ample if $\mathcal{L}^{m}$ is generated by global sections for $m \gg 0$. $\mathcal{L}$ is called ample if it is semi-ample and $\phi_{\mathcal{L}^{m}}$ is an embedding for $m \gg 0$. A line bundle $\mathcal{L}$ on $X$ is called big if the global sections of $\mathcal{L}^{m}$ define a rational map $\phi_{\mathcal{L}^{m}}: X \rightarrow \mathbb{P}^{N}$ such that $X$ is birational to $\phi_{\mathcal{L}^{m}}(X)$ for $m \gg 0$. Note that in this case $\mathcal{L}^{m}$ is not necessarily generated by global sections, so $\phi_{\mathcal{L}^{m}}$ is not necessarily defined everywhere.

A smooth projective variety, $X$, is of general type if $\omega_{X}$ is big. It is easy to see that this condition is invariant under birational equivalence between smooth projective varieties. An arbitrary projective variety is of general type if so is a desingularization of it.

It is expected that varieties of general type are close to being hyperbolic. Lang has made several conjectures describing this phenomenon more concretely [50], Ch. I $\S 3$. One of those conjectures is the following: 
3.5. Lang's Conjecture. Let $X$ be a projective variety. Then $X$ is algebraically hyperbolic if and only if every subvariety of $X$ is of general type.

\section{$\S 4$. VAnishing Theorems}

Vanishing theorems have played a central role in algebraic geometry for the last couple of decades, especially in classification theory. Kollár [34] gives an introduction to the basic use of vanishing theorems as well as a survey of results and applications available at the time. For more recent results one should consult [15], [18], [38], [45], [60]. Because of the availability of those surveys, we will only recall statements that are important for the present article.

In nearly any discussion of vanishing theorems, one must start with the fundamental vanishing theorem of Kodaira.

Theorem 4.1. (Kodaira [33]) Let $X$ be a smooth complex projective variety and $\mathcal{L}$ an ample line bundle on $X$. Then

$$
H^{i}\left(X, \omega_{X} \otimes \mathcal{L}\right)=0 \quad \text { for } \quad i>0 .
$$

This has been generalized in several ways, but as noted above we will restrict to a select few. The original statement of Kodaira was generalized to allow semi-ample and big line bundles in place of ample ones by Grauert and Riemenschneider.

Theorem 4.2. (Grauert-Riemenschneider [22]) Let $X$ be a smooth complex projective variety and $\mathcal{L}$ a semi-ample and big line bundle on $X$. Then

$$
H^{i}\left(X, \omega_{X} \otimes \mathcal{L}\right)=0 \quad \text { for } \quad i>0 .
$$

Remark 4.2.1. "Semi-ample" was later replaced by "nef" in the statement by Kawamata and Viehweg ([29], [63]).

Akizuki and Nakano extended Kodaira's vanishing to include other exterior powers of the sheaf of differential forms:

Theorem 4.3. (Akizuki-Nakano [2]) Let $X$ be a smooth complex projective variety and $\mathcal{L}$ an ample line bundle on $X$. Then

$$
H^{q}\left(X, \Omega_{X}^{p} \otimes \mathcal{L}\right)=0 \quad \text { for } \quad p+q>\operatorname{dim} X .
$$


4.4. Ramanujam [58] gave a simplified proof for (4.3) and showed that it does not hold if one only requires $\mathcal{L}$ to be semi-ample and big.

\section{$\S 5$. Families of Curves}

In this section we are going to give an indication how to prove hyperbolicity for a smooth family of curves over a compact base.

Lemma 5.1. Let $f: X \rightarrow B$ be a smooth family of curves of genus $q \geq 2$. If $\omega_{X / B}$ is ample, then $\omega_{X / B} \otimes f^{*} \omega_{B}^{-1}$ is not ample.

Proof. Since $f$ is smooth then there exists a short exact sequence of locally free sheaves,

$$
0 \rightarrow f^{*} \omega_{B} \rightarrow \Omega_{X} \rightarrow \omega_{X / B} \rightarrow 0
$$

Twisting this short exact sequence by $\omega_{X / B}$, one obtains another one:

$$
0 \rightarrow \omega_{X} \rightarrow \Omega_{X} \otimes \omega_{X / B} \rightarrow \omega_{X / B}^{2} \rightarrow 0
$$

Since $\omega_{X / B}$ is ample, $H^{2}\left(X, \Omega_{X} \otimes \omega_{X / B}\right)=0$ by (4.3). Hence the map $H^{1}\left(X, \omega_{X / B}^{2}\right) \rightarrow H^{2}\left(X, \omega_{X}\right)$ is surjective. The latter group is non-zero, so the former is non-zero as well. However, by (4.1), this implies that indeed $\omega_{X / B} \otimes f^{*} \omega_{B}^{-1}$ cannot be ample.

Exercise 5.2. Let $f: X \rightarrow B$ be a smooth family of curves of genus $q \geq 2$. Prove that if $\omega_{X / B}$ is ample, then $g(B)>1$. Notice that this is a step toward $(\mathbf{H})$. To prove $(\mathbf{H})$, one can proceed in a similar way using more delicate vanishing theorems.

To finish the proof one needs to prove that for a non-isotrivial family $\omega_{X / B}$ is ample. As we will discuss later, this ampleness follows from positivity results on the push-forwards of powers of $\omega_{X / B}$. See $\S 11$ for more details.

To argue that it is actually reasonable to expect that $\omega_{X / B}$ is ample, let us restrict to the case of $g(B)=1$. We are trying to prove that in this case no smooth non-isotrivial $f$ exists. The fibers of $f$ are smooth curves of genus at least two, and the base is of genus one, so $X$ cannot contain any rational curves. Therefore $X$ is a non-ruled minimal surface and thus some power of its canonical bundle is generated by global sections. By adjunction, the 
restriction of $\omega_{X / B}$ to a fiber is the canonical bundle of the fiber, hence it is positive on it. Therefore the morphism induced by the global sections of $\omega_{X / B}$ cannot contract any fibers.

Now there are two possibilities. If the image of this morphism is a curve, then all fibers map isomorphically onto this curve. This implies that the fibers are pairwise isomorphic, contradicting the non-isotriviality of $f$. If the image of the morphism is a surface, then $X$ is of general type and the only way $\omega_{X}$ may not be ample is if it contracts some rational curves. However, we have already established that $X$ does not contain any rational curves, so $\omega_{X}=\omega_{X / B}$ must be ample. This completes the proof of $(\mathbf{H})$ in the case of $g(B)=1$. $\mathbb{P}^{1}$ can be covered by an elliptic curve, hence a smooth family over $\mathbb{P}^{1}$ induces one over the elliptic curve. This implies that no smooth non-isotrivial $f$ exists over $\mathbb{P}^{1}$ either.

\section{§6. Moduli Spaces of Curves}

The notion of parameter spaces was invented in and thus to some extent belongs to the 19th century, a time when varieties were meant to be embedded in projective space and families of varieties were families of embedded varieties.

The 20th century saw a dramatic change in the way we think of varieties. They are no longer subobjects, they exist without a necessary reference to an ambient space. Some of them do not even admit an embedding to projective space, but even those that do are preferred to be viewed abstractly. Correspondingly, we would like to parametrize isomorphism classes without respect to any embedding. For instance, while smooth plane conics are parametrized by a Zariski open subset of $\mathbb{P}^{5}$, they are all isomorphic to $\mathbb{P}^{1}$, so up to isomorphism the parameter space is a single point. Of course, the way to parametrize the possible degree two embeddings of $\mathbb{P}^{1}$ into $\mathbb{P}^{2}$, that is smooth plane conics, should not be forgotten, but in order to follow our principle of regarding varieties in an abstract way we need a corresponding notion of parametrization.

Roughly speaking, a moduli space is a parameter space whose points are in one-to-one correspondence with isomorphism classes of the members of a class of varieties (or schemes). 
Recall that $k$ is an algebraically closed field of characteristic 0 . Let

$$
\mathcal{M}_{q}(k)=\{\text { smooth projective curves of genus } q \text {, defined over } k\} / \simeq \text {. }
$$

By definition, this is only a set, but the theory of moduli tells us that we can make it an algebraic scheme in a natural way:

Theorem 6.1. (Mumford [54]) For $q \geq 2$ there exists a quasi-projective scheme $\mathfrak{M}_{q}$ and a natural bijection between $\mathcal{M}_{q}(k)$ and the set of $k$-points of $\mathfrak{M}_{q}$.

"Natural" here means that every flat family $f: X \rightarrow B$ of smooth projective curves of genus $q$ induces a morphism from the base of the family to $\mathfrak{M}_{q}, \eta_{f}: B \rightarrow \mathfrak{M}_{q}$, such that each point is mapped to the isomorphism class of the fiber over the point, that is, for all $b \in B, \eta_{f}(b)=\left[X_{b}\right]$. A scheme with this property is called a coarse moduli scheme, or a moduli space. The $\mathfrak{M}_{q}$ in the theorem is the moduli space of smooth projective curves of genus $q$.

In order to study compact families, we would like to work with a projective compactification: $\mathfrak{M}_{q} \subseteq \overline{\mathfrak{M}}_{q}$. However, in order for this to be useful, the compactification should also be natural. In other words, it should be the moduli space for an extended class of varieties. This leads us to consider non-smooth families of curves as well.

Definition 6.2. A curve singularity is called a normal crossing if it is locally analytically isomorphic to the singularity of the plane curve $x y=0$. A reduced projective curve $C$ is stable if it has only normal crossing singularities and $\omega_{C}$ is ample.

The extended moduli problem studies

$$
\overline{\mathcal{M}}_{q}(k)=\{\text { stable curves of arithmetic genus } q \text {, defined over } k\} / \simeq
$$

and we have a statement similar to (6.1):

Theorem 6.3. (Deligne-Mumford [13], Knudsen-Mumford [32]) For $q \geq 2$ there exists a projective coarse moduli scheme $\overline{\mathfrak{M}}_{q}$ for $\overline{\mathcal{M}}_{q}$, such that $\mathfrak{M}_{q} \subseteq$ $\overline{\mathfrak{M}}_{q}$ is an open subscheme.

Hence for every family $f: X \rightarrow B$ of stable curves of genus $q$ there exists a morphism $\eta_{f}: B \rightarrow \overline{\mathfrak{M}}_{q}$ such that for all $b \in B, \eta_{f}(b)=\left[X_{b}\right]$. 
6.4. Moduli spaces and Hilbert schemes. The Hilbert scheme, $\operatorname{Hilb}_{p} \mathbb{P}^{N}$, parametrizes subschemes of $\mathbb{P}^{N}$ with fixed Hilbert polynomial $p$. The Hilbert scheme, Hilb $\mathbb{P}^{N}$, parametrizes all subschemes of $\mathbb{P}^{N}$. It is the disjoint union of $\operatorname{Hilb}_{p} \mathbb{P}^{N}$ as $p$ runs through all Hilbert polynomials.

Hilbert schemes belong to the best kind of parameter spaces. There exists a universal family of the schemes parametrized by Hilb $\mathbb{P}^{N}$ such that any family of subschemes of $\mathbb{P}^{N}$ can be obtained from the universal family by pull-back.

Hilbert schemes play a very important role in the construction of moduli spaces. The main idea is the following.

We want to find a parameter space for all smooth (or stable) projective curves of a given genus. The first difficulty is to try to get a hold on all of them at the same time. This problem is solved by a certain boundedness property: For a curve $C$ of genus $q \geq 2$, the sheaf of differential forms, $\omega_{C}$, is an ample line bundle and one can prove that $\omega_{C}^{3}$ is generated by global sections and defines an embedding of $C$ into $\mathbb{P}^{N}$ for $N=5 q-7$. From the construction it is clear that all smooth projective curves of genus $q$ are represented as a curve in $\mathbb{P}^{N}$ with the same Hilbert polynomial. The next step is to verify that the points of the corresponding Hilbert scheme parametrizing our curves form a locally closed subscheme, $\mathcal{H}$, of the ambient Hilbert scheme.

This scheme is still not exactly that we want because the same curve is represented by several points. Even though the sheaf of differentials is unique, giving a universal choice, the embedding defined by the global sections is unique only up to an isomorphism of the target projective space. The morphism depends on the actual generators we choose among the sections. In other words, $\mathrm{PGL}_{n}(k)$ acts on $\mathcal{H}$ and the orbits of this action correspond to the isomorphism classes of smooth projective curves of genus $q$.

Therefore the desired moduli space is given as the $\mathrm{PGL}_{n}(k)$ quotient of $\mathcal{H}$. The fact that this quotient exists as a scheme and has reasonably nice properties is a highly non-trivial fact, but fortunately Mumford's geometric invariant theory tells us that this is the case. Hence the desired moduli space exists.

The naturality of moduli spaces implies that a smooth family of curves of genus $q, f: X \rightarrow B$, induces a map $\eta_{f}: B \rightarrow \mathfrak{M}_{q}$. One might hope that the converse is true, as well, namely that maps into $\mathfrak{M}_{q}$ induce families. In that case families would be in one-to-one correspondence with maps from 
the base of the family to the moduli space and $\mathfrak{M}_{q}$ would be a fine moduli space. This, however, turns out to be too much to ask for.

Of course, this is only the tip of an iceberg. The theory of moduli of curves is a highly developed beautiful subject. The reader is encouraged to continue discovering this topic. A very readable and up to date account is $[25]$.

We now return to Shafarevich's Conjecture and examine the conditions listed in (1.8).

\section{$\S 7$. BOUNDEDNESS}

The aim of this section is to sketch a proof of the following statement.

Theorem 7.1. In order to prove (B), it suffices to show that for some $m>1$ the degree of $f_{*} \omega_{X / B}^{m}$ is bounded, the bound depending on $B \backslash \Delta$ and $q$, but not on $f$.

This section may be hard to digest for the beginner. Anyone willing to accept the above statement should feel free to continue with the next chapter.

Proof. (Sketch) First recall that condition (B) says that admissible families are parametrized by a scheme of finite type.

The naturality of moduli spaces implies that a family $f: X \rightarrow B$ induces a map $\eta_{f}: B \backslash \Delta \rightarrow \mathfrak{M}_{q}$, and since $B$ is a smooth curve, $\eta_{f}$ induces another map $\bar{\eta}_{f}: B \rightarrow \overline{\mathfrak{M}}_{q}$. Unfortunately, as we discussed in the previous section, maps do not necessarily induce families so we cannot parametrize our families this way.

However, we can proceed a different way. One can prove that there exist $m, N>1$ such that for any admissible family $f: X \rightarrow B$, the global sections of $\omega_{X / B}^{m}$ define a map to $\mathbb{P}^{N}$ such that it embeds each smooth fiber of $f$ as a degree $d$ curve in $\mathbb{P}^{N}$. Here $d$ is the degree of $\omega_{X / B}^{m}$ restricted to a fiber of $f$. This induces a map, $\zeta_{f}$, from the base, $B \backslash \Delta$, to the Hilbert scheme of degree $d$ curves in $\mathbb{P}^{N}$, Hilb $\mathbb{P}^{N}$. This Hilbert scheme admits a natural map to $\overline{\mathfrak{M}}_{q}$ and the composition of this natural map with $\zeta_{f}$ is $\eta_{f}$.

This allows one to parametrize admissible families by the maps $\zeta_{f}$ : $B \backslash \Delta \rightarrow \operatorname{Hilb}_{d} \mathbb{P}^{N}$. Maps can be characterized by their graphs. The closure 
of the graph of $\zeta_{f}$ is a curve contained in $B \times \operatorname{Hilb}_{d} \mathbb{P}^{N}$ such that the first projection maps it isomorphically onto $B$. Hence the parameter space we are looking for is a subscheme of the Hilbert scheme of $B \times \operatorname{Hilb}_{d} \mathbb{P}^{N}$ cf. [37].

The Hilbert scheme is an infinite union of schemes of finite type, the components corresponding to the different Hilbert polynominals. The parametrizing scheme being of finite type means that all such graphs should be contained in only finitely many components. That is, there should be only finitely many Hilbert polynomials that can actually occur. All of these curves are isomorphic to $B$ and their image in $\overline{\mathfrak{M}}_{q}$ is non-trivial, so the only relevant coefficient of the Hilbert polynomial is determined by the degree of a fixed ample line bundle pulled back from $\overline{\mathfrak{M}}_{q}$.

Therefore, if $\mathcal{L}$ is a fixed ample line bundle on $\overline{\mathfrak{M}}_{q}$, then to prove $(\mathbf{B})$, it suffices to prove that $\operatorname{deg} \bar{\eta}_{f}^{*} \mathcal{L}$ is bounded on $B$, the bound depending on $B$, $\Delta$ and $q$, but not on $f$. The construction of $\overline{\mathfrak{M}}_{q}$ produces natural ample line bundles: for $p$ sufficiently large and divisible there exist line bundles, $\lambda_{m}^{(p)}$ on $\overline{\mathfrak{M}}_{q}$, such that for a family of stable curves $f: X \rightarrow B$, if $\bar{\eta}_{f}: B \rightarrow \overline{\mathfrak{M}}_{q}$ is the induced map, then

$$
\left(\operatorname{det}\left(f_{*} \omega_{X / B}^{m}\right)\right)^{p}=\bar{\eta}_{f}^{*} \lambda_{m}^{(p)} .
$$

This statement is a consequence of the construction of $\lambda_{m}^{(p)}$ and the fact that one uses the powers of the relative dualizing sheaf to embed the members of the family into projective space. The only missing information we need is that $\lambda_{m}^{(p)}$ is ample for $m>1$. This was proved by Arakelov. Later on we will need the analogous statement for a family of higher dimensional varieties. Fortunately that is also available due to work of Kawamata, Kollár, and Viehweg cf. (9.2). Therefore, to prove (B), it suffices to show that $\operatorname{deg} f_{*} \omega_{X / B}^{m}$ is bounded on $B$ for some $m>1$.

Remark 7.1.1. The notation $\lambda_{m}^{(p)}$ might strike the reader as being odd. The point of this notation is the following. When this line bundle is pulled back to $B$, then it is a $p^{\text {th }}$ power, but originally it may not be. We are considering maps defined by sections of $\left(\operatorname{det}\left(f_{*} \omega_{X / B}^{m}\right)\right)^{p}$ for $m, p$ large and divisible enough. We have no reason to expect this sheaf in general to have any sections for $p=1$, so $\operatorname{det}\left(f_{*} \omega_{X / B}^{m}\right)$ is not expected to be the pull-back of a line bundle from the moduli space. 


\section{§8. Hyperbolicity Revisited}

The first row in the following diagram is the statement of condition $(\mathbf{H})$. The last row shows equivalent conditions for both the assumption and the conclusion.

$$
\begin{aligned}
& 2 g-2+\# \Delta \leq 0 \nexists \quad \nexists f: X \rightarrow B \text { admissible } \\
& \qquad \backslash \Delta=\left\{\begin{array}{cc}
\Downarrow & \nexists B \backslash \Delta \rightarrow \mathfrak{M}_{q} \\
\mathbb{P}^{1} & \text { non-constant, } \\
\mathbb{A}^{1} & \text { induced by } \\
\mathbb{A}^{1} \backslash\{0\} & \text { a family. } \\
\text { elliptic curve }
\end{array}\right.
\end{aligned}
$$

Therefore proving $(\mathbf{H})$ is equivalent to proving that there does not exist a non-constant morphism, induced by a family, of the form $\mathbb{A}^{1} \backslash\{0\} \rightarrow$ $\mathfrak{M}_{q}$ or $E \rightarrow \mathfrak{M}_{q}$, where $E$ is an arbitrary elliptic curve. This resembles hyperbolicity notions we encountered earlier, justifying the name. It is convenient at this point to make a new definition.

Definition 8.1. $\mathfrak{M}_{q}$ is modular hyperbolic if every morphism $B \rightarrow \mathfrak{M}_{q}$ induced by a family is constant for $B=\mathbb{A}^{1} \backslash\{0\}$ or $B$ is abelian variety.

Remark 8.1.1. Essentially, this means that we require the moduli stack to be hyperbolic. For details on stacks see [13], [4], [48].

8.2. The previous observation can be now stated as follows: If $\mathfrak{M}_{q}$ is modular hyperbolic, then $(\mathbf{H})$ holds.

\section{$\S 9$. Weak Boundedness}

In this section we will discuss a property that implies both $(\mathbf{B})$ and $(\mathbf{H})$ and add it to the list of (1.8).

(9.1) Weak Boundedness (WB) For an admissible family $f: X \rightarrow B$, the degree of $f_{*} \omega_{X / B}^{m}$ is bounded above in terms of $g(B), \# \Delta, g\left(X_{\text {gen }}\right)$, $m$. In particular, the bound is independent of $f$.

Using this new notion we can rephrase (7.1): 
Theorem 9.2. $(\mathrm{WB}) \Rightarrow(\mathrm{B})$

The traditional proof of hyperbolicity for curves proceeds via some form of weak boundedness. The key point is that the upper bound obtained on $\operatorname{deg} f_{*} \omega_{X / B}^{m}$ has the form of $(2 g(B)-2+\# \Delta) \cdot c\left(g(B), \# \Delta, g\left(X_{\text {gen }}\right), m\right)$ where $c\left(g(B), \# \Delta, g\left(X_{\text {gen }}\right), m\right)>0$. This proves hyperbolicity; since $\operatorname{det} f_{*} \omega_{X / B}^{m}$ is ample, its degree is positive, so any upper bound of it is positive as well.

In higher dimensions, the bounds obtained are not always in this form. However, perhaps somewhat surprisingly, hyperbolicity follows from the fact of weak boundedness, not from the explicit bound.

Theorem 9.3. $(\mathrm{WB}) \Rightarrow(\mathbf{H})$

A more precise and somewhat more general formulation is the following:

Theorem 9.4. ([46], 0.9) Let $\mathfrak{F}$ be a collection of smooth varieties of general type, $B$ a smooth projective curve and $\Delta \subset B$ a finite subset of $B$. Let

$$
\begin{aligned}
\operatorname{Fam}(B, \Delta, \mathfrak{F})=\{ & f: X \rightarrow B \mid X \text { is smooth, } f \text { is flat and } f^{-1}(t) \in \mathfrak{F} \\
& \text { for all } t \in B \backslash \Delta\} .
\end{aligned}
$$

Assume that there exist $M, m \in \mathbb{N}$ such that for all $(f: X \rightarrow B) \in$ $\mathcal{F} a m(B, \Delta, \mathfrak{F})$

$$
\operatorname{deg}\left(f_{*} \omega_{X / B}^{m}\right) \leq M,
$$

and that $\mathcal{F}$ am $(B, \Delta, \mathfrak{F})$ contains non-isotrivial families. Then $2 g(B)-2+$ $\# \Delta>0$.

Proof. First consider the case $g(B)=1$ and assume that $\Delta=\emptyset$. Let $\tau: B \rightarrow B$ be a finite étale endomorphism of degree $>1$. Then $\left(f_{\tau}\right.$ : $\left.X_{\tau} \rightarrow B_{\tau}\right) \in \mathcal{F} \operatorname{am}(B, \Delta, \mathfrak{F})$ and, by assumption,

$$
\operatorname{deg} \tau \cdot \operatorname{deg}\left(f_{*} \omega_{X / B}^{m}\right)=\operatorname{deg}\left(f_{\tau_{*}} \omega_{X_{\tau} / B_{\tau}}^{m}\right) \leq M .
$$

Since $\operatorname{deg} \tau$ can be arbitrary large and $\operatorname{deg}\left(f_{*} \omega_{X / B}^{m}\right)>0$ by [35], this leads to contradiction.

The case of $g(B)=0, \# \Delta \leq 2$ works similarly. One may assume that $\# \Delta=2$. Again, there exists a finite endomorphism $\tau: B \rightarrow B$ of degree $>1$ such that $\tau$ is smooth over $B \backslash \Delta$ and completely ramified over $\Delta$ : assume that $\Delta=\{0, \infty\}$ and consider the map induced by $z \mapsto z^{n}$. At this point we would like to apply the same argument as above, but before we can do that we have to deal with the singularities this procedure produces. In 
fact we have to start by applying stable reduction to the family ([25], 3.47), and then the singularities we end up working with are sufficiently mild to allow us to use the above argument to finish the proof. For more details see [46], 0.9 .

These statements suggest that (WB) is a condition worth studying.

Next we turn to higher dimensional generalizations. As a first step, one can try to keep the base of the family be a curve and allow higher dimensional fibers. Independently or simultaneously, one can study families over higher dimensional bases. Finally, generalizing the conditions on the fibers naturally leads to the study of families with singular fibers. We will discuss the state of affairs in all of these directions.

\section{§10. Higher Dimensional Fibers}

The first task is to state what we are after, so we need to generalize both the statement and the conditions. The condition that a curve has genus at least 2 , i.e., our assumption that $g\left(X_{\text {gen }}\right) \geq 2$, is equivalent to the condition that $\omega_{X_{\text {gen }}}$ is ample. In higher dimensions, the role of the genus is played by the Hilbert polynomial, so fixing $g\left(X_{\text {gen }}\right)$ will be replaced by fixing $h_{\omega_{X_{\text {gen }}}}$, the Hilbert polynomial of $\omega_{X_{\text {gen }}}$.

10.1. Therefore we have the following starting data:

(10.1.1) a fixed smooth curve $B$ of genus $g$,

(10.1.2) a fixed finite subset $\Delta \subset B$, and

(10.1.3) a fixed polynomial $h$.

Definition 10.2. An admissible family is a non-isotrivial family $f: X \rightarrow$ $B$, such that $X$ is a smooth projective variety and for all $b \in B \backslash \Delta$, the variety $X_{b}$ is smooth and projective with $\omega_{X_{b}}$ ample and $h_{\omega_{X_{b}}}=h$. Two such families are equivalent if they are isomorphic over $B \backslash \Delta$.

Having made this definition, the various parts of Shafarevich's conjecture make sense in any dimension.

\subsection{Higher Dimensional Shafarevich Conjecture.}

(B) Admissible families are parametrized by a scheme $\mathbb{T}$ of finite type.

(R) $\operatorname{dim} \mathbb{T}=0$. 
(H) $\mathbb{T} \neq \emptyset \Rightarrow 2 g-2+\# \Delta>0$.

(WB) For an admissible family $f: X \rightarrow B$, the degree of $f_{*} \omega_{X / B}^{m}$ is bounded in terms of $g, \# \Delta, h$ and $m$.

Next we are going to try to determine which of these statements remain true in higher dimensions.

10.4. $(\mathbf{R})$

Let $Y \rightarrow B$ be an arbitrary non-isotrivial family of curves of genus $\geq 2$, and $C$ a smooth projective curve of genus $\geq 2$. Then $f: X=Y \times C \rightarrow B$ is an admissible family, and a deformation of $C$ gives a deformation of $f$. Therefore (R) fails as stated.

Question 10.5. Under what additional conditions does $(\mathbf{R})$ hold?

10.6. (B), (H), and (WB).

Several results have been obtained in this regard recently.

Migliorini [52] showed that for families of minimal surfaces a somewhat weakened hyperbolicity statement holds, namely that $\delta \geq 1$ if $g \leq 1$. The author showed the same in [39] for families of minimal varieties of arbitrary dimension, later proved $(\mathbf{H})$ for families of minimal surfaces in [40], and then $(\mathbf{H})$ for families of canonically polarized varieties in [43]. Most recently, Viehweg and Zuo [70] proved the analytic version of $(\mathbf{H})$, i.e., Brody hyperbolicity.

Bedulev and Viehweg [7] proved that (B) holds for families of surfaces of general type and that (WB) (and in some cases (B)) holds for families of canonically polarized varieties. As a byproduct of their proof they also obtained that $(\mathbf{H})$ holds in these cases.

Viehweg and Zuo [68] extended (H) and (WB) to families of varieties of general type and of varieties admitting a good minimal model. In [46] the author obtained similar results with different methods allowing the fibers to have rational Gorenstein singularities, but restricting to the case of families of minimal varietes of general type.

(WB) implies (B) as in the curve case cf. [69], 6.2. The proof of (9.4) works in any dimension, so $(\mathbf{W B})$ implies $(\mathbf{H})$ as well. These suggest that (WB) is again the "right" condition to study.

Currently the best known results are the following: 
Theorem 10.7. (Bedulev-Viehweg [7]) Let $f: X \rightarrow B$ be an admissible family with $B, \Delta, h$ fixed. Let $\delta=\# \Delta, g=g(B)$, and $n=\operatorname{dim} X_{\text {gen }}=$ $\operatorname{dim} X-1$. If $f_{*} \omega_{X / B}^{m} \neq 0$, then there exists a positive integer, $e=e(m, h)$ such that

$$
\operatorname{deg} f_{*} \omega_{X / B}^{m} \leq m \cdot e \cdot \operatorname{rk} f_{*} \omega_{X / B}^{m} \cdot(n(2 g-2+\delta)+\delta) .
$$

Theorem 10.8. (Viehweg-Zuo [68], Kovács [46]) A similar statement holds if instead of assuming $X_{\text {gen }}$ smooth and $\omega_{X_{\text {gen }}}$ ample, we assume that $f$ : $X \rightarrow B$ is not birationally isotrivial, and

- [68], [46] $X_{\text {gen }}$ is smooth of general type, or

- [68] $X_{\text {gen }}$ smooth, has a good minimal model and $\kappa\left(X_{\text {gen }}\right) \geq 0$, or

- [46] $X_{\text {gen }}$ has rational Gorenstein singularities and $\omega_{X_{\text {gen }}}$ is ample.

Remark 10.9. One can ask whether the Shafarevich problem holds for families of other type of varieties. There are some known results in this setting as well.

Faltings [19] studied the Shafarevich problem for families of abelian varieties and proved that $(\mathbf{B})$ holds, while $(\mathbf{R})$ fails in general. He also gave an equivalent condition for $(\mathbf{R})$ to hold in this case.

Oguiso and Viehweg [56] considered $(\mathbf{H})$ for families of non-general type surfaces. Their work combined with the previous results show that $(\mathbf{H})$ holds for families of minimal surfaces of non-negative Kodaira dimension.

\section{§11. Positivity of Push-forwards}

One of the most important ingredients in the proofs of the known results is an appropriate variant of a fundamental positivity result.

Definition 11.1. A locally free sheaf, $\mathcal{E}$, is ample if $\mathcal{O}_{\mathbb{P}(\mathcal{E})}(1)$ on $\mathbb{P}(\mathcal{E})$ is ample.

Theorem 11.2. (Kawamata [30], Kollár [35], [36], Viehweg [64], [65]) Let $f: X \rightarrow B$ be an admissible family and $m>1$. If $f_{*} \omega_{X / B}^{m} \neq 0$, then $f_{*} \omega_{X / B}^{m}$ is ample on $B$.

Corollary 11.3. Let $f: X \rightarrow B$ be an admissible family and $m>1$. If $f_{*} \omega_{X / B}^{m} \neq 0$, then $\operatorname{deg} f_{*} \omega_{X / B}^{m}>0$. 
The methods used to prove (11.2) give a more precise estimate of the positivity of these push-forwards:

Theorem 11.4. (Esnault-Viehweg [17], 2.4) Let $f: X \rightarrow B$ be an admissible family, and $\mathcal{M}$ a line bundle on $B$. Assume that there is an $m>1$ such that $\operatorname{deg} \mathcal{M}<\operatorname{deg} f_{*} \omega_{X / B}^{m}$. Set $r=\operatorname{rk} f_{*} \omega_{X / B}^{m}$. Then there exists a positive integer $e=e(m, h)$, such that $\left(f_{*} \omega_{X / B}^{m}\right)^{\otimes e \cdot r} \otimes \mathcal{M}^{-1}$ is ample on $B$.

Corollary 11.5. (for $\Delta=\emptyset$ ) Let $\mathcal{N}$ be a line bundle on $B$ such that $\operatorname{deg} \mathcal{N}^{m \cdot e \cdot r}<\operatorname{deg} f_{*} \omega_{X / B}^{m}$. Then $\omega_{X / B} \otimes f^{*} \mathcal{N}^{-1}$ is ample on $X$.

Proof. (Sketch) Since $\left(f_{*}\left(\omega_{X / B}^{m} \otimes f^{*} \mathcal{N}^{-m}\right)\right)^{\otimes e \cdot r} \simeq\left(f_{*} \omega_{X / B}^{m}\right)^{\otimes e \cdot r} \otimes \mathcal{N}^{-m \cdot e \cdot r}$, (11.4) implies that $f_{*}\left(\omega_{X / B}^{m} \otimes f^{*} \mathcal{N}^{-m}\right)$ is ample on $B$. Furthermore, $\omega_{X / B}^{m} \otimes$ $\left.f^{*} \mathcal{N}^{-m}\right|_{X_{\text {gen }}} \simeq \omega_{X_{\text {gen }}}^{m}$ is ample on $X_{\text {gen }}$. Hence $\omega_{X / B} \otimes f^{*} \mathcal{N}^{-1}$ is ample both "horizontally" and "vertically", so it is ample. For details about the last step see [46], 7.6.

This allows us to reduce the proof of (WB) to finding an appropriate line bundle:

11.6. Plan for proving (WB):

- Find an $\mathcal{N}$ depending only on $B$ and $\Delta$ such that $\omega_{X / B} \otimes f^{*} \mathcal{N}^{-1}$ is not ample on $X$.

- Then by (11.5) we have that $\operatorname{deg} \mathcal{N}^{m \cdot e \cdot r} \nless \operatorname{deg} f_{*} \omega_{X / B}^{m}$. In other words $\operatorname{deg} f_{*} \omega_{X / B}^{m} \leq m \cdot e \cdot r \cdot \operatorname{deg} \mathcal{N}$.

11.7. We will find such an $\mathcal{N}$ using vanishing theorems. The main idea is the following: we want to find a line bundle such that twisting with the relative dualizing sheaf does not yield an ample line bundle. Ample line bundles appear in many vanishing theorems, so one way to prove that a given line bundle is not ample is to prove that a cohomology group does not vanish that would if the line bundle were ample. Next we are going to look at the needed vanishing theorems. 


\section{§12. Logarithmic Vanishing Theorems}

In order to proceed we will need more delicate vanishing theorems than before. Our starting point is the theorem of Esnault and Viehweg that extends (4.3) to sheaves of logarithmic differential forms:

Theorem 12.1. (Esnault-Viehweg [17], 6.4) Let $X$ be a smooth complex projective variety and $\mathcal{L}$ an ample line bundle on $X$. Further let $D$ be a normal crossing divisor on $X$. Then

$$
H^{q}\left(X, \Omega_{X}^{p}(\log D) \otimes \mathcal{L}\right)=0 \quad \text { for } \quad p+q>\operatorname{dim} X .
$$

12.2. Extending the known vanishing theorems in a different direction, Navarro-Aznar et al. proved a version of the Kodaira-Akizuki-Nakano vanishing theorem for singular varieties that implies our previous statements: (4.1), (4.2), and (4.3) cf. [55] in [22]. We will return to this statement later.

As mentioned earlier, in order to prove (WB), we need a suitable vanishing theorem. The following is a somewhat weaker statement than that is really needed, but shows the main idea of the proof and how to apply it.

Theorem 12.3. [40], [43] Let $f: X \rightarrow B$ be a family such that $B$ is a smooth projective curve. Assume that $D=f^{*} \Delta$ is a normal crossing divisor. Let $n=\operatorname{dim} X_{\text {gen }}$ and $\mathcal{L}$ an ample line bundle on $X$ such that $\mathcal{L} \otimes f^{*} \omega_{B}(\Delta)^{-n}$ is also ample. Then

$$
H^{n+1}\left(X, \mathcal{L} \otimes f^{*} \omega_{B}(\Delta)\right)=0 .
$$

Proof. After taking exterior powers of the sheaves of logarithmic differential forms, one has the following short exact sequence for each $p=1, \ldots, n+1$ :

$$
0 \longrightarrow \Omega_{X / B}^{p-1}(\log D) \otimes f^{*} \omega_{B}(\Delta) \longrightarrow \Omega_{X}^{p}(\log D) \longrightarrow \Omega_{X / B}^{p}(\log D) \longrightarrow 0 .
$$

Define $\mathcal{L}_{p}=\mathcal{L} \otimes f^{*} \omega_{B}(\Delta)^{1-p}$ for $p=0, \ldots, n+1$. Then the above short exact sequence yields:

$0 \longrightarrow \Omega_{X / B}^{p-1}(\log D) \otimes \mathcal{L}_{p-1} \longrightarrow \Omega_{X}^{p}(\log D) \otimes \mathcal{L}_{p} \longrightarrow \Omega_{X / B}^{p}(\log D) \otimes \mathcal{L}_{p} \longrightarrow 0$.

$\mathcal{L}_{p}$ is ample for $p=1, \ldots, n+1$, since either $\omega_{B}(\Delta)$ or $\omega_{B}(\Delta)^{-1}$ is nef, so by $(12.1) H^{n+1-(p-1)}\left(X, \Omega_{X}^{p}(\log D) \otimes \mathcal{L}_{p}\right)=0$ (recall that $\left.\operatorname{dim} X=n+1\right)$. Hence the map

$$
H^{n+1-p}\left(X, \Omega_{X / B}^{p}(\log D) \otimes \mathcal{L}_{p}\right) \longrightarrow H^{n+1-(p-1)}\left(X . \Omega_{X / B}^{p-1}(\log D) \otimes \mathcal{L}_{p-1}\right)
$$


is surjective for $p=1, \ldots, n+1$. Observe that these maps form a chain as $p$ runs through $p=n+1, n, \ldots, 1$. Hence the composite map

$$
H^{0}\left(X, \Omega_{X / B}^{n+1}(\log D) \otimes \mathcal{L}_{n+1}\right) \longrightarrow H^{n+1}\left(X, \mathcal{L}_{0}\right)
$$

is also surjective. However, $\Omega_{X / B}(\log D)$ is of rank $n$, so $\Omega_{X / B}^{n+1}(\log D)=0$, and therefore $H^{n+1}\left(X, \mathcal{L}_{0}\right)=H^{n+1}\left(X, \mathcal{L} \otimes f^{*} \omega_{B}(\Delta)\right)=0$ as well.

12.4. We are finally able to prove (WB), at least for $\Delta=\emptyset$, by combining positivity and vanishing: (11.3) and (11.5) with $\mathcal{N}=\mathcal{O}_{B}$ imply that $\omega_{X / B}$ is ample. Since

$$
H^{n+1}(X, \underbrace{\omega_{X / B} \otimes f^{*} \omega_{B}}_{\omega_{X}}) \neq 0,
$$

this and (12.3) imply that $\omega_{X / B} \otimes f^{*} \omega_{B}^{-n}$ cannot be ample. Then (11.5) with $\mathcal{N}=f^{*} \omega_{B}^{n}$ implies that

$$
\operatorname{deg} f_{*} \omega_{X / B}^{m} \leq \operatorname{deg} f^{*} \omega_{B}^{n \cdot m \cdot e \cdot r}=m \cdot e \cdot r \cdot \operatorname{dim} X_{\text {gen }} \cdot(2 g-2) .
$$

Remark 12.4.1. For a complete proof of (WB) without the assumption $\Delta=\emptyset$, see [7], [46], or [69].

Next we will look at other generalizations.

\section{§13. Smooth Fibers that are Minimal of General Type AND Singular Fibers}

Viehweg and Zuo [69], respectively Kovács [46] present different approaches to this case. Here we discuss the latter. For a survey on the former, the reader is referred to Viehweg [67].

The following principle has been applied with great success in birational geometry.

Principle 13.1. Studying an ample line bundle on a singular variety is similar to studying a semi-ample and big line bundle on a smooth variety. 
13.2. The usual situation is the following. Our goal is to prove a statement for a pair, $(X, \mathcal{L})$, where $X$ is possibly singular, and $\mathcal{L}$ is ample on $X$. Instead of working on $X$ we work on a desingularization $f: Y \rightarrow X$, and consider the semi-ample and big line bundle $\mathcal{K}=f^{*} \mathcal{L}$. A prominent example of this trick is the use of the Kawamata-Viehweg vanishing theorem in the Minimal Model Program.

13.3. Here we will turn the situation upside-down. Our goal is a statement for $(Y, \mathcal{K})$, where $Y$ is smooth and $\mathcal{K}$ is semi-ample and big on $Y$. Instead of working on $Y$ we construct a pair $(X, \mathcal{L})$ and a map $f: Y \rightarrow X$, where $X$ is possibly singular, $\mathcal{L}$ is ample on $X, f$ is birational, and $\mathcal{K}=f^{*} \mathcal{L}$.

The motivation for this approach is that we would like to extend the results to the case when $\omega_{X_{\text {gen }}}$ is only semi-ample and big. One crucial ingredient of the proof is an appropriate version of the Kodaira-AkizukiNakano vanishing theorem (4.3). However, as Ramanujam (4.4) pointed out, (4.3) fails if the line bundle in question is only assumed to be semiample and big. On the other hand, Navarro-Aznar et al. proved a singular version (12.2), so one hopes that this way the proof can be made to work.

13.4. Derived categories. In order to state the singular version of the Kodaira-Akizuki-Nakano vanishing theorem, we need to use derived categories. The reader unfamiliar with the basics may wish to consult [26] and [9] for definitions and details.

A derived category is essentially the unified approach to deal with resolutions and derived functors simultaneously. The following dictionary shows a correspondence between derived categorical notions and what they generalize.

$\begin{array}{ccc}\text { THIS } & \rightsquigarrow & \text { GENERALIzes THIS } \\ \text { "complex" } & \rightsquigarrow & \text { "sheaf" } \\ \text { "hypercohomology" } & \rightsquigarrow & \text { "cohomology" } \\ \mathbb{H}^{i}(X, A) & \rightsquigarrow & H^{i}(X, A) \\ \text { "distinguished triangle" } & \rightsquigarrow & \text { "short exact sequence" } \\ A \longrightarrow B \longrightarrow C \stackrel{+1}{\longrightarrow} & \rightsquigarrow & 0 \rightarrow A \rightarrow B \rightarrow C \rightarrow 0\end{array}$

13.5. Du Bois's complex. We also need Du Bois's generalized De Rham complex. The original construction of Du Bois's complex, $\Omega_{X}(\log D)$, is based on simplicial resolutions. The reader interested in the details is referred to the original article [14]. Note also that a simplified construction 
was later obtained in [23] via the general theory of cubic resolutions. An easily accessible introduction can be found in [61].

The word "hyperresolution" will refer to either simplicial or cubic resolution. Formally, the construction of $\underline{\Omega}_{X}(\log D)$ is the same regardless which resolution is used and no specific aspects of either resolution will be used.

The following definition is included to make sense of the statements of some of the forthcoming theorems. It can be safely ignored if the reader is not interested in the detailed properties of Du Bois's complex and is willing to accept that it is a very close analogue of the De Rham complex of smooth varieties.

Definition 13.6. Let $X$ be a complex scheme and $D$ a closed subscheme whose complement in $X$ is dense. Then $(X ., D.) \rightarrow(X, D)$ is a good hyperresolution if $X . \rightarrow X$ is a hyperresolution, and if $U .=X . \times_{X}(X \backslash D)$ and $D$. $=X$. \U., then $D_{i}$ is a divisor with normal crossings on $X_{i}$ for all $i$.

Let $X$ be a complex scheme of dimension n. Let $D_{\text {filt }}(X)$ denote the derived category of filtered complexes of $\mathcal{O}_{X}$-modules with differentials of order $\leq 1$ and $D_{\text {filt,coh }}(X)$ the subcategory of $D_{\text {filt }}(X)$ of complexes $K$, such that for all $i$, the cohomology sheaves of $G r_{\text {filt }}^{i} K$ are coherent cf. [14], [23]. Let $D(X)$ and $D_{\text {coh }}(X)$ denote the derived categories with the same definition except that the complexes are assumed to have the trivial filtration. The superscripts,,$+- b$ carry the usual meaning (bounded below, bounded above, bounded). Isomorphism in these categories is denoted by $\simeq_{\text {qis }}$. If $K^{\cdot}$ is a complex in any of the above categories, then $h^{i}\left(K^{\cdot}\right)$ denotes the $i$-th cohomology sheaf of $K$. In particular, every sheaf is naturally a complex with $h^{i}=0$ for $i \neq 0$.

The right derived functor of an additive functor $F$, if it exists, is denoted by $R F$ and $R^{i} F$ is short for $h^{i} \circ R F . \mathbb{H}^{i}, \mathbb{H}_{Z}^{i}$, and $\mathcal{H}_{Z}^{i}$ denote $R^{i} \Gamma, R^{i} \Gamma_{Z}$, and $R^{i} \mathcal{H}_{Z}$ respectively, where $\Gamma$ is the functor of global sections, $\Gamma_{Z}$ is the functor of global sections with support in the closed subset $Z$, and $\mathcal{H}_{Z}$ is the functor of local sections with support in the closed subset $Z$. Note that according to this terminology, if $\phi: Y \rightarrow X$ is a morphism and $\mathcal{F}$ is a coherent sheaf on $Y$, then $R \phi_{*} \mathcal{F}$ is the complex whose cohomology sheaves give the usual higher direct images of $\mathcal{F}$.

Theorem 13.7. (Du Bois [14], 6.3, 6.5) Let $X$ be a proper complex scheme of finite type and $D$ a closed subscheme whose complement is dense in $X$. 
Then there exists a unique $\underline{\Omega}_{X}(\log D) \in \mathrm{Ob}\left(D_{\text {filt }}(X)\right)$ with the following properties, using the notation:

$$
\underline{\Omega}_{X}^{p}(\log D):=G r_{\text {filt }}^{p} \underline{\Omega}_{X}(\log D)[p] .
$$

(13.7.1) Let $j: X \backslash D \rightarrow X$ be the inclusion map. Then

$$
\underline{\Omega}_{X}(\log D) \simeq_{\mathrm{qis}} R j_{*} \mathbb{C}_{X \backslash D} .
$$

(13.7.2) $\underline{\Omega}_{(-)}(\log (-))$ is functorial, i.e., if $\phi: Y \rightarrow X$ is a morphism of proper complex schemes of finite type, then there exists a natural map $\phi^{*}$ of filtered complexes

$$
\phi^{*}: \underline{\Omega}_{X}(\log D) \rightarrow R \phi_{*} \underline{\Omega}_{Y}\left(\log \phi^{*} D\right)
$$

Furthermore, $\underline{\Omega}_{X}(\log D) \in \mathrm{Ob}\left(D_{\text {filt,coh }}^{b}(X)\right)$ and if $\phi$ is proper, then $\phi^{*}$ is a morphism in $D_{\text {filt,coh }}^{b}(X)$.

(13.7.3) Let $U \subseteq X$ be an open subscheme of $X$. Then

$$
\left.\underline{\Omega}_{X}(\log D)\right|_{U} \simeq_{\mathrm{qis}} \underline{\Omega}_{U}\left(\left.\log D\right|_{U}\right) .
$$

(13.7.4) There exists a spectral sequence degenerating at $E_{1}$ and abutting to the singular cohomology of $X \backslash D$ :

$$
E_{1}^{p q}=\mathbb{H}^{q}\left(X, \underline{\Omega}_{X}^{p}(\log D)\right) \Rightarrow H^{p+q}(X \backslash D, \mathbb{C}) .
$$

(13.7.5) If $\varepsilon .:(X ., D.) \rightarrow(X, D)$ is a good hyperresolution, then

$$
\underline{\Omega}_{X}(\log D) \simeq_{\text {qis }} R \varepsilon_{\cdot *} \Omega_{X .}(\log D .) .
$$

In particular, $h^{i}\left(\underline{\Omega}_{X}^{p}(\log D)\right)=0$ for $i<0$.

(13.7.6) There exists a natural map, $\mathcal{O}_{X} \rightarrow \underline{\Omega}_{X}^{0}(\log D)$, compatible with (13.7.2).

(13.7.7) If $X$ is smooth and $D$ is a normal crossing divisor, then

$$
\underline{\Omega}_{X}(\log D) \simeq_{\text {qis }} \Omega_{X}(\log D) .
$$

In particular,

$$
\underline{\Omega}_{X}^{p}(\log D) \simeq_{\text {qis }} \Omega_{X}^{p}(\log D) .
$$

(13.7.8) If $\phi: Y \rightarrow X$ is a resolution of singularities, then

$$
\underline{\Omega}_{X}^{\operatorname{dim} X}(\log D) \simeq_{\mathrm{qis}} R \phi_{*} \omega_{Y}\left(\phi^{*} D\right) .
$$

Naturally, one may choose $D=\emptyset$ and then it is simply omitted from the notation. The same applies to $\underline{\Omega}_{X}^{p}:=G r_{\text {filt }}^{p} \underline{\Omega}_{X}[p]$. We are now able to state the singular version of the Kodaira-Akizuki-Nakano vanishing theorem. 
Theorem 13.8. (Navarro-Aznar [55], [23]) Let $X$ be a complex projective variety and $\mathcal{L}$ an ample line bundle on $X$. Then

$$
\mathbb{H}^{q}\left(X, \underline{\Omega}_{X}^{p} \otimes \mathcal{L}\right) \quad \text { for } \quad p+q>\operatorname{dim} X .
$$

Since Du Bois's complex agrees with the De Rham complex for smooth varieties, this theorem reduces to the Kodaira-Akizuki-Nakano theorem in the smooth case. However, this theorem is still not strong enough in our original situation if $\Delta \neq \emptyset$. We need a singular version of Esnault-Viehweg's logarithmic vanishing theorem (12.1).

Theorem 13.9. [46] Let $X$ be a complex projective variety and $\mathcal{L}$ an ample line bundle on $X$. Further let $D$ be a normal crossing divisor on $X$. Then

$$
\mathbb{H}^{q}\left(X, \underline{\Omega}_{X}^{p}(\log D) \otimes \mathcal{L}\right)=0 \quad \text { for } \quad p+q>\operatorname{dim} X .
$$

To adapt the proof of (WB) to the singular case we need a singular version of (12.3). Besides the above vanishing theorem we also need an analogue of the sheaf of relative logarithmic differentials.

Theorem-Definition 13.10. [46] Let $f: X \rightarrow B$ be a morphism between complex varieties such that $\operatorname{dim} X=n+1$ and $B$ is a smooth curve. Let $\Delta \subseteq B$ be a finite set and $D=f^{*} \Delta$. For every nonnegative integer $p$ there exists a complex $\underline{\Omega}_{X / B}^{p}(\log D) \in \mathcal{O} b j(D(X))$ with the following properties.

(13.10.1) The natural map $\wedge_{p}$ factors through $\underline{\Omega}_{X / B}^{p}(\log D) \otimes f^{*} \omega_{B}(\Delta)$, i.e., there exist maps:

$$
\begin{aligned}
& w_{p}^{\prime \prime}: \underline{\Omega}_{X}^{p}(\log D) \otimes f^{*} \omega_{B}(\Delta) \rightarrow \underline{\Omega}_{X / B}^{p}(\log D) \otimes f^{*} \omega_{B}(\Delta) \quad \text { and } \\
& w_{p}^{\prime}: \underline{\Omega}_{X / B}^{p}(\log D) \otimes f^{*} \omega_{B}(\Delta) \rightarrow \underline{\Omega}_{X}^{p+1}(\log D)
\end{aligned}
$$

such that $\wedge_{p}=w_{p}^{\prime} \circ w_{p}^{\prime \prime}$.

If $w_{p}=w_{p}^{\prime \prime} \otimes i d_{f^{*} \omega_{B}(\Delta)^{-1}}: \underline{\Omega}_{X}^{p}(\log D) \rightarrow \underline{\Omega}_{X / B}^{p}(\log D)$, then

$\underline{\Omega}_{X / B}^{p}(\log D) \otimes f^{*} \omega_{B}(\Delta) \stackrel{w_{p}^{\prime}}{\longrightarrow} \underline{\Omega}_{X}^{p+1}(\log D) \stackrel{w_{p+1}}{\longrightarrow} \underline{\Omega}_{X / B}^{p+1}(\log D) \stackrel{+1}{\longrightarrow}$

is a distinguished triangle in $D(X)$. 
(13.10.3) $w_{p}$ is functorial, i.e., if $\phi: Y \rightarrow X$ is a $B$-morphism, then there are natural maps in $D(X)$ forming a commutative diagram:

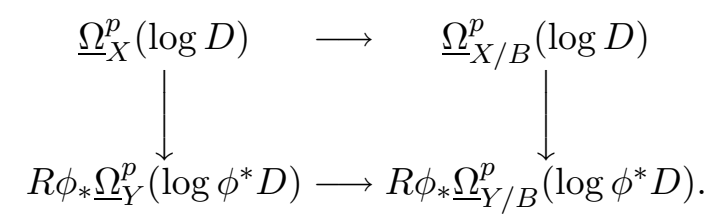

(13.10.4) If $f$ is smooth over $B \backslash \Delta$, then $\underline{\Omega}_{X / B}^{p}(\log D) \simeq_{\mathrm{qis}} \Omega_{X / B}^{p}(\log D)$.

(13.10.5) $\underline{\Omega}_{X / B}^{r}(\log D)=0$ for $r>n$ and if $f$ is proper, then $\underline{\Omega}_{X / B}^{p}(\log D) \in$ $\mathcal{O} b j\left(D_{\text {coh }}^{b}(X)\right)$ for every $p$.

Using these objects one can make the proof work to obtain the following theorem. It is in a non-explicit form. For more precise statements see [46], (7.8), (7.10), (7.11), (7.13).

Theorem 13.10. Fix $B, \Delta \subset B$. Then weak boundedness holds for families of canonically polarized varieties with rational Gorenstein singularities over $B \backslash \Delta$ with fixed Hilbert polynomial admitting a simultaneous resolution of singularities. In particular, $2 g-2+\# \Delta>0$ for these families by (9.4).

As a corollary, one obtains weak boundedness for non-birationallyisotrivial families of minimal varieties of general type.

\section{$\S 14$. Higher Dimensional Bases}

The remaining generalization is to allow $B$ to have arbitrary dimension. Let $B$ be a smooth projective variety, $\Delta \subset B$ a divisor with normal crossings and $h$ a polynomial. The definition of an admissible family is formally the same as in (10.2).

By [66], there exists a coarse moduli scheme, $\mathfrak{M}_{h}$, parametrizing such $X_{b}$ 's. Hence for an admissible family, $f: X \rightarrow B$, there exists an associated map, $\eta_{f}: B \backslash \Delta \rightarrow \mathfrak{M}_{h}$, defined by $b \mapsto\left[X_{b}\right]$.

Since $B$ is now allowed to be higher dimensional, the notion of isotriviality is no longer the best one to consider. Observe that $f$ is isotrivial if and only if $\eta_{f}$ is constant. Saying that $f$ is not isotrivial would allow the family to be isotrivial in certain directions. What we want to assume is that the family "truly" changes in any direction on $B$. To express this we define the family's variation in moduli. 
Definition 14.1. (Viehweg [64], [65], Kollár [35]) $\operatorname{Var} f:=\operatorname{dim}(\operatorname{Im} \eta) \leq$ $\operatorname{dim} B$.

We are interested in the case $\operatorname{Var} f=\operatorname{dim} B$. In (8.2), we observed that hyperbolicity follows if we know that $\mathfrak{M}_{h}$ is modular hyperbolic. In fact, for hyperbolicity over a 1-dimensional base, we only needed the corresponding property of $\mathfrak{M}_{h}$ for curves. However, we would also like to know that every morphism $A \rightarrow \mathfrak{M}_{h}$ induced by a family is constant, where $A$ is an arbitrary abelian variety. This follows from the next theorem.

Theorem 14.2. [41], [43] $\mathfrak{M}_{h}$ is modular hyperbolic.

Remark 14.2.1. This statement also follows from boundedness by an argument similar to the one used in the proof of (9.4).

As before, this implies that if $f: X \rightarrow \mathbb{P}^{1}$ is an admissible family, then $\# \Delta>2$. More generally, for an admissible family $f: X \rightarrow \mathbb{P}^{m}$ with $\operatorname{Var} f=m$, this implies that $\operatorname{deg} \Delta>2$. However, we expect that in this case deg $\Delta$ should be larger than $m+1$.

Theorem 14.3. (Viehweg-Zuo [69]) deg $\Delta>m+1$, i.e., $\omega_{\mathbb{P}^{m}}(\Delta)$ is ample.

Remark 14.3.1. Viehweg and Zuo actually prove a lot more than this in [69]. Please see the article for details.

It is now natural to suspect that a more general statement should hold. The following statement to this effect is part of a more general conjecture of Viehweg [67].

14.4. Viehweg's Conjecture. If $f: X \rightarrow B$ is an admissible family, then $\omega_{B}(\Delta)$ is big.

This is known to be true for families of curves by [67], 2.6 and for $B=\mathbb{P}^{n}$ and various other cases by [69]. However, this question is far from being completely settled. 


\section{REFERENCES}

[1] U. Angehrn and Y.-T. Siu, Effective freeness and point separation for adjoint bundles, Invent. Math., 122 (1995), 291-308.

[2] Y. Akizuki and S. Nakano, Note on Kodaira-Spencer's proof of Lefschetz theorems, Proc. Jap. Acad., 30 (1954), 266-272.

[3] S. Arakelov, Families of algebraic curves with fixed degeneracies, Izv. A. N. SSSR, 35 (1971), 1269-1293.

[4] M. Artin, Versal deformations and algebraic stacks, Invent. Math., 27 (1974), 165189.

[5] W. Barth, C. Peters and A. Van de Ven, Compact Complex Surfaces, Ergebnisse der Mathematik und ihrer Grenzgebiete (3), vol. 4., Springer (1984).

[6] A. Beauville, Le nombre minimal de fibres singulières d'une courbe stable sur $\mathbb{P}^{1}$, Astérisque, 86 (1981), 97-108.

[7] E. Bedulev and E. Viehweg, On the Shafarevich conjecture for surfaces of general type over function fields, Invent. Math., 139 (2000), 603-615.

[8] F. Catanese and M. Schneider, Polynomial bounds for abelian groups of automorphisms, Compositio Math., 97 (1995), 1-15.

[9] B. Conrad, Grothendieck Duality and Base Change, LNM 1750, Springer (2000).

[10] M. A. A. de Cataldo, Vanishing via lifting to second Witt vectors and a proof of an isotriviality result, J. Algebra, 219 (1999), 255-265.

[11] M. de Franchis, Un teorema sulle involuzioni irrazionali, Rend. Circ. Mat. Palermo, 36 (1913), 368. See also in "Collected works of Michele de Franchis", Edited by C. Ciliberto and E. Sernesi., Rend. Circ. Mat. Palermo (2) Suppl., 27 (1991), 523.

[12] A. J. de Jong and F. Oort, On extending families of curves, J. Algebraic Geom., 6 (1997), 545-562.

[13] P. Deligne and D. Mumford, The irreducibility of space of curves of given genus, Publ. Math. IHES, 36 (1969), 75-110.

[14] Ph. Du Bois, Complex de De Rham filtré d'une variété singulière, Bull. Soc. Math. France, 109 (1981), 41-81.

[15] L. Ein, Multiplier ideals, vanishing theorems and applications, in: Algebraic Geometry, Santa Cruz 1995 (S. Bloch et al., eds.), Proc. Symp. Pure Math., vol. 62 (1997), pp. 203-219.

[16] H. Esnault and E. Viehweg, Logarithmic de Rham complexes and vanishing theorems, Invent. Math., 86 (1986), 161-194.

[17] H. Esnault and E. Viehweg, Effective bounds for semipositive sheaves and for the height of points on curves over complex function fields, Compositio Math., $\mathbf{7 6}$ (1990), 69-85.

[18] H. Esnault and E. Viehweg, Lectures on vanishing theorems, DMV Seminar, vol. 20, Birkhäuser (1992). 
[19] G. Faltings, Arakelov's Theorem for abelian varieties, Invent. Math., 73 (1983), $337-348$.

[20] G. Faltings, Endlichkeitssätze für abelsche Varietäten über Zahlkörpern, Invent. Math., 73 (1983), 349-366.

[21] J.-M. Fontaine, Il n'y a pas de variété abélienne sur $\mathbb{Z}$, Invent. Math., 81 (1985), $515-538$.

[22] H. Grauert and O. Riemenschneider, Verschwindungssätze für analytische Kohomologiegruppen auf komplexen Räumen, Invent. Math., 11 (1970), 263-292.

[23] F. Guillén, V. Navarro-Aznar, P. Pascual-Gainza and F. Puerta, Hyperrésolutions cubiques et descente cohomologique, LNM 1335, Springer (1988).

[24] J. Harris, Algebraic Geometry: A First Course, Graduate Texts in Mathematics, vol. 133, Springer (1992).

[25] J. Harris and I. Morrison, Moduli of curves, Graduate Texts in Mathematics, vol. 187, Springer (New York, 1998).

[26] R. Hartshorne, Residues and Duality, LNM 20, Springer (1966).

[27] R. Hartshorne, On the De Rham cohomology of algebraic varieties, Publ. Math. IHES, 45 (1975), 5-99.

[28] R. Hartshorne, Algebraic Geometry, Graduate Texts in Mathematics, vol. 52, Springer (1977).

[29] Y. Kawamata, A generalisation of Kodaira-Ramanujam's vanishing theorem, Math. Ann., 261 (1982), 43-46.

[30] Y. Kawamata, Kodaira dimension of algebraic fiber spaces over curves, Invent. Math., 66 (1982), 57-71.

[31] Y. Kawamata, K. Matsuda and K. Matsuki, Introduction to the Minimal Model Problem, in: Algebraic Geometry (Sendai, 1985) (T. Oda, ed.), Adv. Stud. Pure Math., vol. 10, Kinokuniya - North-Holland (1987), pp. 283-360.

[32] F. F. Knudsen and D. Mumford, The projectivity of the moduli space of stable curves. I. Preliminaries on "det" and "Div", Math. Scand., 39 (1976), 19-55.

[33] K. Kodaira, On a differential geometric method in the theory of analytic stacks, Proc. Nat. Acad. USA, 39 (1953), 1268-1273.

[34] J. Kollár, Vanishing theorems for cohomology groups, in: Algebraic Geometry Bowdoin 1985, Proc. Symp. Pure Math., vol. 46 (1987), pp. 233-243.

[35] J. Kollár, Subadditivity of the Kodaira dimension: Fibers of general type, in: Algebraic Geometry (Sendai, 1985), (T. Oda, ed.), Advanced Studies in Pure Math., vol. 10, Kinokuniya-North Holland (1987), pp. 361-398.

[36] J. Kollár, Projectivity of Complete Moduli, J. Diff. Geom., 32 (1990), 235-268.

[37] J. Kollár, Rational Curves on Algebraic Varieties, Ergebnisse der Mathematik und ihrer Grenzgebiete (3), vol. 32, Springer (1996).

[38] J. Kollár, Singularities of pairs, in: Algebraic Geometry Santa Cruz 1995, Proc. Symp. Pure Math., vol. 62 (1997), pp. 221-287. 
[39] S. J. Kovács, Smooth families over rational and elliptic curves, J. Algebraic Geom., 5 (1996), 369-385.

[40] S. J. Kovács, On the minimal number of singular fibers in a family of surfaces of general type, J. reine angew. Math., 487 (1997), 171-177.

[41] S. J. Kovács, Families over a base with a birationally nef tangent bundle, Math. Ann., 308 (1997), 347-359.

[42] S. J. Kovács, Relative De Rham complexes for non-smooth morphisms, in: Birational Algebraic Geometry (Y. Kawamata, V. Shokurov eds.), Contemp. Math., vol. 207 (1997), pp. 89-100.

[43] S. J. Kovács, Algebraic hyperbolicity of fine moduli spaces, J. Algebraic Geom., 9 (2000), 165-174.

[44] S. J. Kovács, A characterization of rational singularities, Duke Math. J., 102 (2000), 187-191.

[45] S. J. Kovács, Rational, log canonical, Du Bois singularities II: Kodaira vanishing and small deformations, Compositio Math., 121 (2000), 297-304.

[46] S. J. Kovács, Logarithmic vanishing theorems and Arakelov-Parshin boundedness for singular varieties, Compositio Math., 131 (2002), 291-317.

[47] S. J. Kovács, Vanishing theorems, boundedness and hyperbolicity over higher dimensional bases, Proc. AMS (to appear).

[48] G. Laumon and L. Moret-Bailly, Champs algébriques, Ergebnisse der Mathematik und ihrer Grenzgebiete (3), vol. 39, Springer (2000).

[49] S. Lang, Introduction to complex hyperbolic spaces, Springer (1987).

[50] S. Lang, Survey of Diophantine Geometry, Springer (1997).

[51] Ju. I. Manin, Rational points on algebraic curves over function fields, Izv. Akad. Nauk SSSR Ser. Mat., 27 (1963), 1395-1440.

[52] L. Migliorini, A smooth family of minimal surfaces of general type over a curve of genus at most one is trivial, J. Algebraic Geom., 4 (1995), 353-361.

[53] L. Moret-Bailly, Un théorème de pureté pour les familles de courbes lisses, C.R. Acad. Sci. Paris Sér. I Math., 300 (1985), 489-492.

[54] D. Mumford, Geometric invariant theory, Ergebnisse der Mathematik und ihrer Grenzgebiete, vol. 34, Springer (1965).

[55] V. Navarro-Aznar, Théorèmes d'annulation, in: Hyperrésolutions cubiques et descente cohomologique, LNM 1335, Springer (1988), pp. 133-160.

[56] K. Oguiso and E. Viehweg, On the isotriviality of families of elliptic surfaces, $J$. Algebraic Geom., 10 (2001), 569-598.

[57] A. Parshin, Algebraic curves over function fields, Izv. A.N. SSSR, 32 (1968), 11451170.

[58] C. P. Ramanujam, Remarks on the Kodaira vanishing theorem, J. Indian Math. Soc., 36 (1972), 41-51. 
[59] V. V. Shokurov, Letters of a bi-rationalist I. A projectivity criterion, in: Birational Algebraic Geometry, (Y. Kawamata, V. Shokurov, eds.), Contemp. Math., vol. 207 (1997), pp. 143-152.

[60] K. E. Smith, Vanishing, singularities and effective bounds via prime characteristic local algebra, in: Algebraic Geometry Santa Cruz 1995, Proc. Symp. Pure Math., vol. 62 (1997), pp. 289-325.

[61] J. H. M. Steenbrink, Vanishing theorems on singular spaces, Astérisque, 130 (1985), 330-341.

[62] S. L. Tan, The minimal number of singular fibres of a semistable curve over $\mathbb{P}^{1}$, J. Algebraic Geom., 4 (1995), 591-596.

[63] E. Viehweg, Vanishing theorems, J. reine angew. Math., 335 (1982), 1-8.

[64] E. Viehweg, Weak positivity and the additivity of Kodaira dimension for certain fibre spaces, in: Algebraic Varieties and Analytic Varieties, Advanced Studies in Pure Math., vol. 1, North-Holland (1983), pp. 329-353.

[65] E. Viehweg, Weak positivity and the additivity of the Kodaira dimension II., in: Classification of algebraic and analytic manifolds, Progress in Math., vol. 39, Birkhäuser (1983), pp. 567-590.

[66] E. Viehweg, Quasi-Projective Moduli of Polarized Manifolds, Ergebnisse der Mathematik und ihrer Grenzgebiete (3), vol. 30, Springer (1995).

[67] E. Viehweg, Positivity of direct image sheaves and applications to families of higher dimensional manifolds (2000) Lecture notes, "School on Vanishing Theorems and Effective Results in Algebraic Geometry", ICTP, Trieste.

[68] E. Viehweg and K. Zuo, On the isotriviality of families of projective manifolds over curves, J. Algebraic Geom., 10 (2001), 781-799.

[69] E. Viehweg and K. Zuo, Base spaces of non-isotrivial families of smooth minimal models, Complex geometry (Göttingen, 2000), 279-328, Springer (Berlin, 2002).

[70] E. Viehweg and K. Zuo, On the Brody hyperbolicity of moduli spaces for canonically polarized manifolds, preprint.

[71] Q. Zhang, Global holomorphic one-forms on projective manifolds with ample canonical bundles, J. Alg. Geom., 6 (1997), 777-787.

Sándor J. Kovács

Department of Mathematics

University of Washington

Seattle, WA 98195

U.S.A.

kovacs@math. washington.edu 